\title{
Refining the use of oxygen consumption as a proxy corrosion rate measure for archaeological and historic iron
}

\author{
N. J. Emmerson ${ }^{\mathrm{a}} \mathbb{D}$, J. H. Seifert ${ }^{\mathrm{b}} \mathbb{D}$, D. E. Watkinson ${ }^{\mathrm{c}} \mathbb{( D}$ \\ Department of Archaeology and Conservation, School of History, Archaeology, and Religion, Cardiff \\ University, Cardiff, Wales, UK
}

Received: 7 November 2020 / Accepted: 22 April 2021

(C) The Author(s) 2021

\begin{abstract}
Since its introduction to the field of heritage science, oxygen consumption has found multiple applications in the assessment of artefact stability and the examination of conservation treatment efficiencies. Early papers identified various factors affecting oxygen measurements and produced preliminary data on their effects. This paper follows on from the early research by other workers and presents guidance on the application of oxygen consumption as a proxy corrosion rate measurement based on a decade of experience at Cardiff University. It examines the rationale for key aspects of the Cardiff protocols for oxygen consumption and presents data to underpin these. Finally, it provides instructional guidance for heritage scientists adopting oxygen consumption for their research applications.
\end{abstract}

\section{Introduction}

Developing strategies for preserving heritage objects and structures relies on understanding the reaction of materials with their ambient environment and controlling this. While preservation aims to prevent change entirely, this remains either largely unachievable or prohibitively expensive to attain. Therefore, determining the rate of change of a material, as a function of its environment, is essential for identifying its current and on-going decay. Acting as a predictor of longevity and informing risk assessment, this rate of change feeds into management processes and determines strategies for preserving heritage.

From marine contexts, through burial environments to exposure in the atmosphere, oxygen supports many decay processes including biodeterioration, polymer oxidation and metallic corrosion. Consequently, quantitative measurement of oxygen consumption can be used to predict ongoing decay rates and to inform the design of treatments and evaluate their effectiveness. The development of technology that can record oxygen concentration remotely through a transparent medium offered an inexpensive and apparently simple method for recording oxygen consumption of a material within a sealed container [1]. For the first time, it was

\footnotetext{
a e-mail: emmersonnj@ cardiff.ac.uk (corresponding author)

be-mail: seifertjh@cardiff.ac.uk

c e-mail: watkinson@ cardiff.ac.uk
} 
possible to record the reaction rate of an object as a function of oxygen consumed and the environment within the container housing it.

Initial reporting identified a wide range of environmental and practical variables that impacted on the collection of data using this method [2,3]. The need for sensitive remote recording of oxygen concentration and its impact on procedure and management in heritage is particularly evident for the preservation of heritage metals. Thickett et al. [4] investigated corrosion rates of unstable archaeological iron and copper alloys to inform approaches to their display. Interpreting the effectiveness of treatments followed. For decades, highly unstable salt contaminated objects from archaeological deposits had been treated by desalination methods [5-7]. These aimed to induce stabilisation by removing electrolytes, but success was a subject of hot debate, which was largely informed by anecdotal reporting of post-treatment physical change $[8,9]$. Understanding of treatment outcomes has been transformed by the oxygen sensing technique, which has enabled treatment design [10] to be linked to treatment success $[11,12]$. Similarly, quantitative measurement of post-excavation corrosion rates [13] has produced more granular understanding of the practical application of desiccation for preserving archaeological iron using microclimates [14]. The performance of protective coatings and surface preparation techniques on wrought iron has also been assessed and is used by practitioners to guide their treatments $[15,16]$.

Oxygen consumption has been employed in Conservation Science at Cardiff University since 2010 as a proxy measurement of corrosion rate for heritage metals, where it replaced the use of dynamic recording of mass gain due to ongoing corrosion [17-20]. It has been used to examine corrosion rates of archaeological and analogous wrought and cast iron and copper alloy samples, mild steel and finely divided powders. Based on the Matthiesen method [1-3], the Cardiff application of the technique has been refined over its decade of application to ferrous metal corrosion studies. Numerous publications [11, 13, 15, 21-24] have reported the method used but have not discussed in detail the rationale behind the selection of parameters for the hardware, environmental factors and oxygen meter settings.

Aim and objectives: The aim of this paper is to offer advice on refinement of the method for using oxygen consumption within sealed vessels as a proxy corrosion rate measurement for heritage metals, particularly iron. To do this, experiences of the following methodological variables are discussed:

Oxygen sensing equipment;

Data outputs and conversion calculations;

Choice of reaction vessels;

Preparation of samples;

Oxygen consumption of system components;

Internal and external environmental parameters;

Fibre optic probe optimisation;

Measurement protocol;

Calculating loss of metal through corrosion.

\section{Oxygen sensing principles}

The principle of oxygen consumption as a proxy for corrosion rate measurement is based on the dynamic quenching of luminescence by oxygen molecules. Fluorescent substances can be excited by energy which is then emitted as light or can be transferred to other molecules, in which case their fluorescence is quenched [25]. Most luminophores are quenched by 


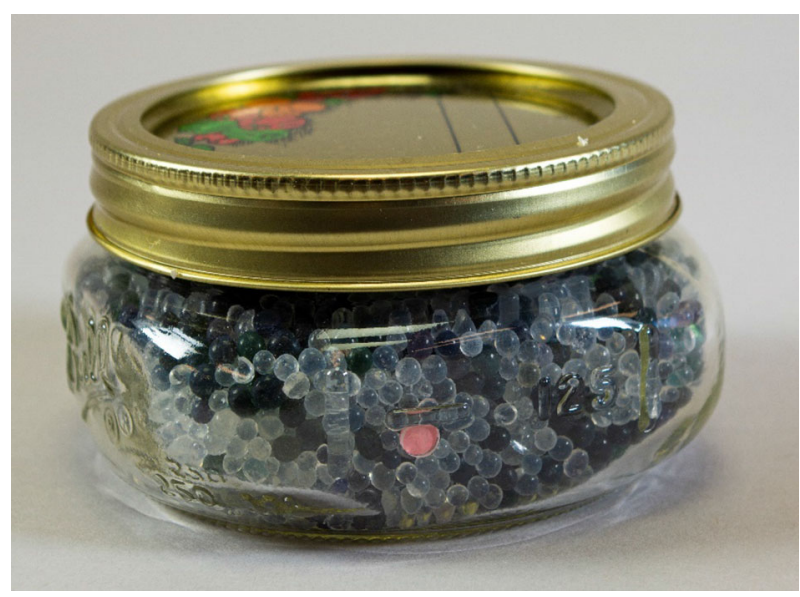

Fig. 1 A $250 \mathrm{ml}$ Mason Ball jar used as an oxygen consumption monitoring vessel

molecular oxygen in a process resulting from collisional encounters between the two, known as dynamic quenching [26]. This process has been described as follows [27]:

- A luminophore (L) is excited by a pulse of light at an appropriate wavelength.

- The excited luminophore $\left(\mathrm{L}^{*}\right)$ relaxes to its ground state either by light emission or by a non-radiative route.

- Luminescence intensity decays exponentially over time with the rate of decay characterised as the luminescence lifetime.

- Oxygen can quench fluorescence by collision with luminophores in a process which transfers energy to the oxygen molecule.

$$
L *+O_{2} \rightarrow L+O_{2} *
$$

- The effect of this radiationless relaxation is a reduction in the intensity and lifetime of the luminescence in the presence of oxygen.

- As the quenching behaviour of the oxygen is near-ideal, the extent to which it occurs can be related directly to the pressure of available oxygen.

In the systems described here, an oxygen-sensitive ruthenium complex that is excited by light of $505 \mathrm{~nm}$ wavelength is fixed to a polymer base to produce sensor spots of $5 \mathrm{~mm}$ diameter [1]. The sensor spot is attached to the inside of an airtight vessel within which is sealed a sample and silica gel to control the internal humidity (Figs. 1, 2, 3). The oxygen pressure inside the vessel can be read through its transparent wall using a meter (see Sect. 3) that produces light pulses at the correct wavelength and measures the fluorescent light returned from the sensor spot. If the sample consumes oxygen over time, this can be detected in subsequent readings as an increase in fluorescence intensity and lifetime caused by a decrease in oxygen pressure.

\section{Equipment}

At Cardiff, initial experiments used the World Precision Instruments OxyMini AOT and subsequently the PreSens (Precision Sensing) Fibox 4 then OXY-1 SMA and OXY-4 SMA 


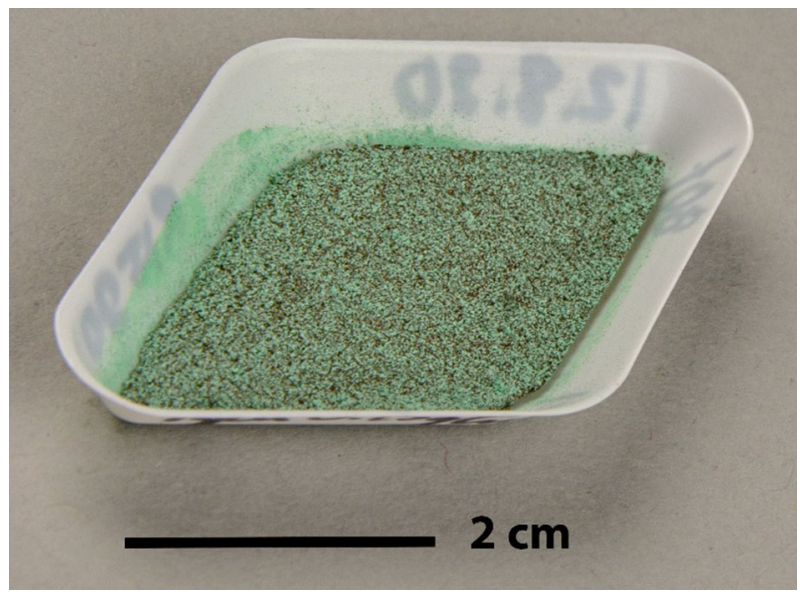

Fig. 2 A copper corrosion powder sample in a weighboat. The sample is then placed inside a $250 \mathrm{ml}$ Mason Ball reaction vessel with silica gel (Fig. 3) for oxygen consumption analysis

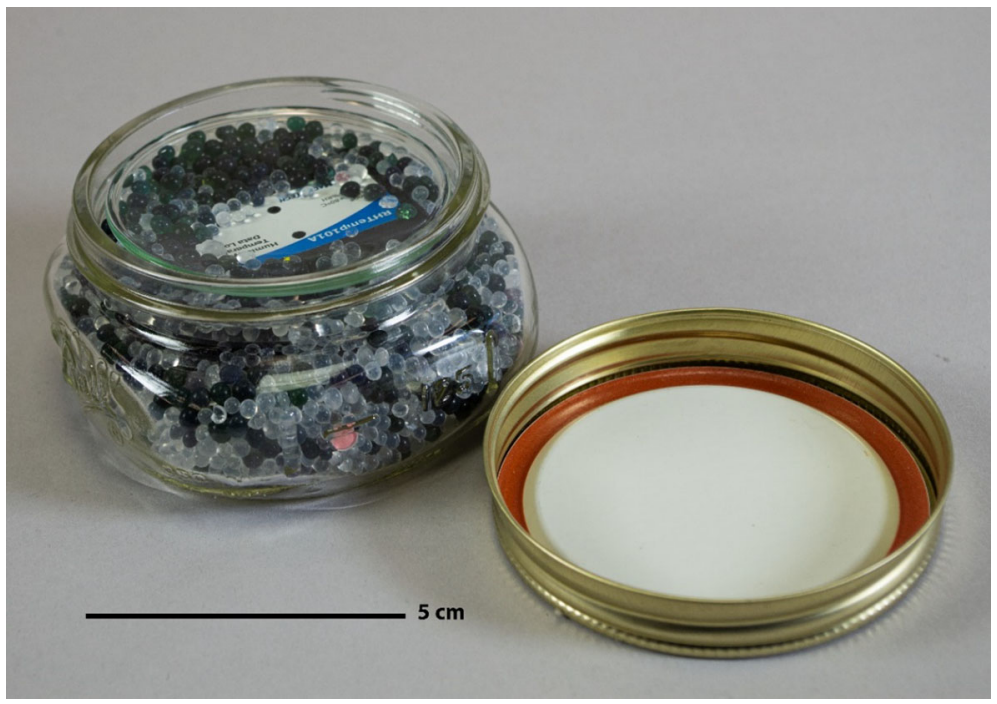

Fig. 3 A $250 \mathrm{ml}$ Mason Ball reaction vessel, filled with conditioned silica gel. Note the placement of the MadgeTech 101A environmental monitor and watchglass barrier on which the sample sits

meters. All systems have employed PSt3 type sensor spots. The technical specifications for the current equipment are given in Table 1.

\section{Data outputs and converting oxygen pressure}

The data output from the Measurement Studio software is a measurement of oxygen as a partial pressure of the gas within the reaction vessel. As any consumption of oxygen will be relative to the vessel volume and influenced by temperature, to allow data to be comparable 
Table 1 Specifications of PreSens OXY-1 SMA and OXY-4 SMA meters and sensor spots [28] currently in use at Cardiff

\begin{tabular}{|c|c|}
\hline \multicolumn{2}{|l|}{ Meter } \\
\hline Meter types & PreSens OXY-1 SMA; OXY-4 SMA \\
\hline Temperature sensor & Pt100 \\
\hline Temperature sensor performance range & $0-50{ }^{\circ} \mathrm{C}$; Resolution $\pm 0.1^{\circ} \mathrm{C} ;$ Accuracy $\pm 1.0^{\circ} \mathrm{C}$ \\
\hline Temperature range: operation / storage & $0-50{ }^{\circ} \mathrm{C} /-20-70{ }^{\circ} \mathrm{C}$ \\
\hline Relative humidity operating range & $0-80 \% \mathrm{RH}$ \\
\hline Software & PreSens Measurement Studio \\
\hline \multicolumn{2}{|l|}{ Sensor } \\
\hline Sensor type & PSt 3 for gaseous and dissolved $\mathrm{O}_{2}$ \\
\hline Measurement range & $0-100 \% \mathrm{O}_{2} ; 0-1000 \mathrm{hPa}$ \\
\hline Limit of detection & $0.03 \%$ oxygen \\
\hline \multirow[t]{4}{*}{ Resolution } & $\pm 0.01 \% \mathrm{O}_{2}$ at $0.21 \% \mathrm{O}_{2}$ \\
\hline & $\pm 0.1 \% \mathrm{O}_{2}$ at $20.9 \% \mathrm{O}_{2}$ \\
\hline & $\pm 0.1 \% \mathrm{hPa}$ at $2 \mathrm{hPa}$ \\
\hline & $\pm 1 \% \mathrm{hPa}$ at $207 \mathrm{hPa}$ \\
\hline Accuracy & $\pm 0.4 \% \mathrm{O}_{2}$ at $20.9 \% \mathrm{O}_{2} ; \pm 0.05 \% \mathrm{O}_{2}$ at $0.2 \% \mathrm{O}_{2}$ \\
\hline Measurement temperature range & $0-50{ }^{\circ} \mathrm{C}$ \\
\hline Response time & $<6 s$ \\
\hline $\mathrm{pH}$ tolerance range & pH 1-14 \\
\hline Calibration & $\begin{array}{l}\text { Two-point calibration in oxygen-free environment and } \\
\text { air-saturated environment }\end{array}$ \\
\hline Storage stability & $\begin{array}{l}5 \text { years at room temperature in dry conditions with exclusion of } \\
\text { light }\end{array}$ \\
\hline \multicolumn{2}{|l|}{ Optical fibre } \\
\hline Optical fibre type & Polymer Optical Fibre (POF) \\
\hline Dimensions & Optical diameter $2 \mathrm{~mm}$ (2.8 mm including cladding) \\
\hline Temperature stability range & Resistant to temperatures up to $+70^{\circ} \mathrm{C}$ \\
\hline
\end{tabular}

between investigations it is converted to the moles or mass of oxygen consumed by the sample using the ideal gas law:

$$
P V=n R T \text { or } n=P V / R T
$$

where $\mathrm{P}=$ pressure of gas (atm.), $\mathrm{V}=$ volume of the gas (l), $\mathrm{n}=$ amount of substance (moles), $\mathrm{R}=$ ideal gas constant, $\mathrm{T}=$ temperature $(\mathrm{K})$.

Calculating the volume of gas (V) in a flexible reaction container can be achieved by introducing a gas by injection once the container and its contents are set up [29]; however, it can be more difficult for a rigid reaction vessel. The empty volume of a vessel can be calculated by measuring the volume of liquid that it holds. The volume is then reduced by each component within the vessel. The volume of a sample or component can be calculated from its dimensions for a simple shape or from its mass for a sample of a single material using the density of that material. However, in the case of a complex shape or dissimilar materials (e.g. an iron core covered in voluminous corrosion products), it can be difficult to calculate sample volume. Where samples can be immersed in a liquid, this can be achieved by displacement. In other cases, it may be necessary to estimate the volume, acknowledging 
the impact this may have on the resulting data. Silica gel volume can be estimated from the mass of silica gel and its density, taking into account that silica gel conditioned to different humidities contains different volumes of water (see Sect. 8.1). The importance of calculating the amount of oxygen consumed in this way is also shown in investigation of the influence of temperature (see Sect. 8.2).

\section{Choice of reaction vessels}

The selection of a suitable reaction vessel for oxygen consumption corrosion rate measurement is complex. There are several factors which dictate the suitability of vessels for the purpose, which must:

Be transparent to allow unhindered transmission of light between the external probe and internal sensor spot.

Be as airtight as possible to create a closed system.

Be a suitable size (aperture and volume) to house the sample and any humidity buffer material, such as silica gel.

Be rigid (for preference-see Sect. 8.4).

Not consume or evolve oxygen itself.

Although other workers have succeeded in producing closed systems using flexible barrier material (e.g. Escal ${ }^{\mathrm{TM}}$ ) [1-3, 29], the influence of barometric pressure on the oxygen pressure readings within vessels sealed with a flexible barrier [3] (see Sect. 8.4) encouraged the Cardiff method to focus on sourcing vessels with rigid lids, the gas pressures within which are less likely to be subject to fluctuations in ambient pressure. Initial testing at Cardiff identified as suitable Mason Ball jars with two-part steel lids comprising a plate with deformable rubber sealing gasket and a sealing ring that screws onto the body of the vessel (Fig. 1; Table 2). These are available in volumes of $250 \mathrm{~cm}^{3}$ to $3000 \mathrm{~cm}^{3}$ and have been used with silica gel to condition the internal vessel environment (see Sect. 8.1) [11, 13, 15, 22, 30].

The refractive properties of glass mean that vessel wall thickness may affect the pathway of light between the optical fibre tip and the sensor. To mitigate this, the same vessel type is used for all samples, the sensor spot is placed where the vessel wall has minimal curvature

Table 2 Oxygen ingress into $250 \mathrm{ml}$ Mason Ball jars filled with nitrogen gas and sealed with a flat disc and sealing ring mechanism (data after Watkinson et al. [13])

\begin{tabular}{lll}
\hline Reaction Vessel & Oxygen ingress (mbar/day) & Oxygen ingress (mg/day) \\
\hline 1 & 0.0077 & 0.0025 \\
2 & 0.0132 & 0.0043 \\
3 & 0.0137 & 0.0045 \\
4 & 0.0130 & 0.0043 \\
5 & 0.0132 & 0.0043 \\
6 & 0.0153 & 0.0050 \\
7 & 0.0142 & 0.0047 \\
8 & 0.0026 & 0.0009 \\
Average & 0.0116 & 0.0038
\end{tabular}


and always in the same position on each vessel using a template. The angle of the probe to optical fibre to the wall of the vessel is also constant at $90^{\circ}$.

Matthiesen [3] recommends reducing the headspace (free air volume) within a reaction vessel to obtain results more quickly for slow reactions, as the same consumption of oxygen molecules by a reaction will have a greater impact on the pressure of oxygen relative to other gases in the vessel. The Cardiff method controls humidity within the vessel using silica gel (see Sect. 8.1) which occupies much of the free space, although there is air within and between the silica gel beads. The headspace is then reduced further by the sample. The time taken to obtain a measurable rate of oxygen consumption for a sample is variable dependent on the tendency of the samples to corrode in the vessel environment, i.e. at a given humidity. Artefacts of broadly similar form from the same site can have very different corrosion rates with some depleting all oxygen within a vessel in $48 \mathrm{~h}$ and others depleting oxygen much more slowly. To reduce measurement duration, an inert material could be added to the vessel to occupy headspace, for example a block of glass. The volume of silica gel would be reduced to accommodate this.

Ideally, leakage rates of vessels should be calculated with the same headspace as during the oxygen consumption measurements, but this is difficult to achieve practically as the sample cannot be included in any control measurements and the diffusion of air out of pore spaces in the silica gel may artificially inflate oxygen ingress rates.

\section{Preparation of samples}

One of the key advantages of this corrosion rate measurement system is its flexibility to accommodate a range of sample types and operate relatively non-destructively. Cardiff research has measured oxygen consumption of heritage artefacts, analogous samples, and powders to examine corrosion rates in a range of environments.

\subsection{Heritage artefacts}

Non-destructive analysis of artefacts is the holy grail of conservation and heritage science. When managed carefully, oxygen consumption measurement can provide that real-time, nondestructive assessment of corrosion rates and use of heritage artefacts is encouraged. This is not without difficulties, however, which can include:

- Obtaining sufficient numbers of similar artefacts to provide statistically viable results, depending on the nature of the study.

- Sourcing appropriately sized vessels to accommodate the artefacts (see Sect. 5 and sealing method).

- Measuring the surface area (of the metal core) and overall volume of samples for corrosion rate calculations.

Successful research projects have used Roman wrought iron nails from different archaeological sites in Britain [11, 13]. The ubiquity of these objects on Roman sites, their broadly similar physical form and their limited value for archaeological study makes them an ideal sample set for oxygen consumption. As with any artefacts covered with corrosion products, it is important to x-ray the samples to establish that they contain a metal core prior to oxygen consumption measuring.

For some heritage materials, it may be possible to sample a large artefact destructively to produce consistent samples of a suitable size for oxygen consumption testing within a 
vessel. This has been achieved with historic wrought iron plate in studies at Cardiff $[15,16]$ and offers an option for testing on large numbers of reasonably standardised historic material samples with minor localised compositional differences.

\subsection{Analogues}

Where there is no possibility of testing heritage artefacts for ethical or practical reasons, or where a greater degree of standardisation between samples is required than can be provided by heritage material, analogous samples may be used. These offer greater flexibility in size and form and can be useful for comparison studies, but consideration must be given to the extent to which they reflect the corrosion rates of heritage materials in practice.

\subsection{Coated samples}

Many corrosion prevention strategies, particularly for exterior heritage metalwork exposed to atmospheric corrosion, rely on application of protective coatings. The efficacy of these coatings can be examined using oxygen consumption which measures the consumption of the underlying metal $[15,31]$. Methodologies employing this technique must consider the oxygen consumption of the coating material which can influence the change in oxygen pressure within the vessel.

To account for this, a simple technique involves creating analogous samples of a material which does not consume oxygen and coating these using the same protocol as the coating of the samples. This has been used successfully with glass substrates of the same dimensions as the sample [30]. Recording the mass of the samples and glass substrates as each coating is applied allows verification that the coatings have been applied similarly in both cases. Any consumption of the coated glass samples can be subtracted from the consumption of the coated samples to allow for calculation of the consumption due to corrosion. To minimise the likelihood of coatings consuming oxygen, manufacturer instructions regarding curing or drying time for coatings should be followed and the requisite amount of time allowed between application of the coatings and sealing of the samples within the reaction vessels.

\subsection{Finely divided powders}

Although the use of heritage samples may present the most realistic data for understanding corrosion rates of artefacts, there are instances in which aspects of treatments or corrosion mechanisms may be investigated using finely divided powders. The large surface area of these powders and the ability to vary the proportions of the reactants allows for substantial quantities of data to be collected in a short space of time. An example of its application has been in the study at Cardiff of akaganéite $(\beta-\mathrm{FeOOH})$, the chloride bearing corrosion product which is responsible for much of the post-excavation corrosion of archaeological iron [32, 33]. The influence of aqueous washing treatments for heritage artefacts on the ability of $\beta$-FeOOH to corrode iron has been investigated in this way [23].

The method for carrying out these tests has involved grinding together in an agate pestle and mortar the required masses of the reactant powders, for example iron powder and $\beta$ $\mathrm{FeOOH}$. These are then contained within a polyethylene weighboat (Fig. 2) and placed on a bed of silica gel conditioned to the required relative humidity (see Sect. 8.1) within the reaction vessel (see Placement within the reaction vessel) and the vessel sealed. The rate of these reactions usually requires that the oxygen within the vessel be measured continuously 
(see Sect. 10). The use of finely divided powders does not account for the corrosion dynamics of an artefact but can inform on some fundamental aspects of relative corrosion rates.

\subsection{Placement within the reaction vessel}

To control and vary the relative humidity within a reaction vessel (see Sect. 8.1), the vessels contain silica gel conditioned to the relevant relative humidity. Where possible, samples are placed on top of this bed of silica gel with a separator between the samples and silica gel. For most samples, a watchglass or polyethylene weighboat is appropriate for this. A datalogger which records the environment within the vessel to ensure that it maintained the required environmental parameters is also included, embedded in the silica gel. An example of this configuration can be seen in Fig. 3.

\section{Oxygen consumption of system components}

As the accurate measurement of corrosion rates relies on being able to measure oxygen consumption by the sample, it is important to consider any other components within the reaction vessel that may consume oxygen. To do this, control oxygen consumption studies should be carried out on vessels containing the same physical components but without the samples. For example, oxygen concentrations in Mason Ball vessels containing silica gel, weighboat or watchglass, and a datalogger have been recorded to ensure that none of the system components consume oxygen themselves. Results [11,34] show that there is no net oxygen consumption by these components and any consumption recorded in vessels set up in this way with metal samples can be attributed to the samples themselves. This concept is similar to the measurement of oxygen consumption by coatings (see Sect. 6.3) and should form part of a suite of control experiments designed to isolate the consumption of the samples from any other influences on the oxygen levels within a vessel, which includes ingress of oxygen by leakage.

\section{Internal and external environmental parameters}

Environmental parameters may influence the oxygen measurements recorded within a reaction vessel in two ways. They may affect the rate at which oxygen is consumed by the sample, and they may influence the value for oxygen that the meter records at any point in time.

\subsection{Relative humidity}

It is well known that the corrosion rate of metals is influenced by relative humidity and numerous studies have been conducted to examine this [12, 13, 35]. This is important for two main reasons. First, oxygen consumption provides an excellent method for studying this effect as the relative humidity within a reaction vessel can be controlled using silica gel or solutions (saturated salts or glycerol) that do not consume oxygen themselves $[1,11,35]$. Second, this highlights the importance of ensuring that the relative humidity is controlled to a static value, even where the influence of relative humidity on corrosion rates is not under study, to ensure comparability between datasets. Relative humidity is also intimately linked to temperature in an inverse relationship which means that control of temperature also becomes an important factor (see Sect. 8.2). 
Table 3 Increase in mass of silica gel as conditioned to $20-90 \%$ relative humidities [36]

\begin{tabular}{lll}
\hline Relative humidity (\%) & Mass increase (\%) & $\begin{array}{l}\text { Example for } \\
250 \mathrm{ml} \text { Mason } \\
\text { Ball vessels }(\mathrm{g})\end{array}$ \\
\hline 20 & - & 130.0 \\
30 & 104.4 & 135.7 \\
40 & 109.6 & 142.5 \\
50 & 115.2 & 149.7 \\
60 & 119.8 & 155.7 \\
70 & 121.7 & 158.2 \\
80 & 122.5 & 159.2 \\
90 & 123.2 & 160.1 \\
\hline
\end{tabular}

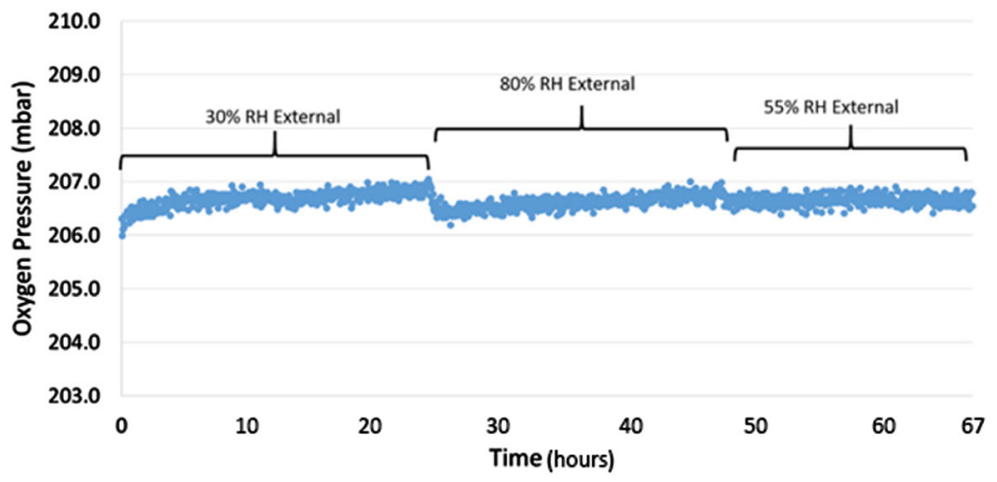

Fig. 4 The effect of changing external relative humidity $(\mathrm{RH})$ on the oxygen pressure as measured for a sealed $250 \mathrm{ml}$ Mason Ball vessel containing only air. The raw oxygen pressure is presented as all other variables remain constant

The Cardiff method uses conditioned silica gel to maintain humidities, other workers offer methods for use of solutions to achieve this [21] [30]. To ensure consistency in volume of silica gel, a scale of increasing mass for the same volume of silica gel according to its relative humidity has been calculated according to its capacity to hold water molecules (Table 3) [36]. This allows the same volume of silica gel to be included in each vessel which is important in relation to calculating results from raw data (see Sect. 11) although the additional volume of water within the silica gel beads at increasing humidity must also be accounted for in calculations.

It is easy to see why the relative humidity inside a reaction vessel is important to a rigorous experimental protocol. Anecdotally, it has been noticed that changes in external RH can affect the ability to attain the required amplitude (see Sect. 9), therefore affecting oxygen readings. This is less easy to understand and has been investigated by varying the RH inside a climate chamber at static $20^{\circ} \mathrm{C}$ whilst continually logging the internal oxygen pressure within a sealed $250 \mathrm{ml}$ Mason Ball vessel containing only air at ambient atmospheric pressure (Fig. 4).

Although a change in oxygen pressure is measured, this is small and difficult to explain. The temperature remained constant at $20^{\circ} \mathrm{C}$ during this time, as confirmed by dataloggers within the climate chamber, therefore is not the cause of the observed phenomenon. Rigorous logging of the temperature change when the climate chamber increases humidity (achieved by effectively boiling water to generate steam) has shown that a $0.05^{\circ} \mathrm{C}$ temperature increase 
is the maximum observed. Therefore, it is unlikely that this can be attributed to temperature change. It is possible that changes in the relative humidity of the measurement environment affect the path of light between the tip of the fibre optic cable and the wall of the reaction vessel. This has been recorded for distances of $1 \mathrm{~mm}$ between optical fibres in transmitted light pathways; an increase in the light power received was observed with increasing humidity in the air space between fibres [37].

\subsection{Temperature}

As with relative humidity, temperature has multiple effects on the oxygen consumption measurement process and, unlike external relative humidity, the external temperature has a direct influence on the internal environment of a vessel. First, the temperature has an indirect relationship with relative humidity; a rise or fall in temperature is expected to correlate with a decrease or increase in relative humidity within a vessel, respectively. The use of buffer material such as silica gel aims to limit this effect and saturated salt, or glycerol solutions are known to create very stable relative humidities despite temperature changes [35]. Second, temperature influences the rate of reactions and changes in temperature may affect the rate of oxygen consumption during corrosion accordingly. This effect, and the ability of oxygen consumption to measure this, is currently under study at Cardiff. Third, increasing temperature increases molecular energy which will increase gas pressure within a sealed system according to the ideal gas law (Eq. 2) and may influence the rate of oxygen exchange between the internal and external environment of a vessel. Finally, the optical measurement of oxygen is temperature dependent [27]. It is therefore important to control and record temperature during measurement.

Figure 5 shows the influence of temperature on the raw oxygen pressure measured within a sealed empty $250 \mathrm{ml}$ Mason Ball vessel containing air at ambient pressure and housed in a climatic chamber controlling the external relative humidity (to static 50\% $\mathrm{RH}$ ) and varying temperature over time as indicated. It can be seen that temperature influences the oxygen pressure reading, with pressure increasing with temperature $20-30{ }^{\circ} \mathrm{C}$ causing an increase of c. 8 mbar, reducing from $30-25^{\circ} \mathrm{C}$ sees a reduction of c. 3.5 mbar and a reduction from $25-22.5^{\circ} \mathrm{C}$ sees a reduction of c. 2.5 mbar. Using the ideal gas law to convert the oxygen pressure into oxygen mass, the temperature effects are largely compensated for in the calculation. However, as the temperature fluctuates, short-term changes in the oxygen mass are seen.

Although the fluctuations in oxygen mass are small, when measuring relatively slow corrosion rates, it may be expected that fluctuating temperatures during logging will affect the oxygen pressure values that are obtained and argues for maintaining constant temperature at least while logging the oxygen pressure even if the reaction itself is not thought to be influenced by temperature. The Cardiff method includes stabilisation of temperature in the initial set-up process for vessels. The contents of the vessel are brought to the logging temperature before sealing to ensure that the pressure of air within the vessel is not affected after sealing by any change in temperature. The temperature of the vessels is maintained in a climate chamber throughout an investigation which also has the effect of excluding ambient light.

\subsection{Ambient light}

The oxygen measurement method relies on luminescence, with molecules in the sensor spot excited by light delivered from the meter via the fibre optic cable and emitted light returned from the spot to the meter, again via the fibre optic [1]. Given this relationship with light, it 


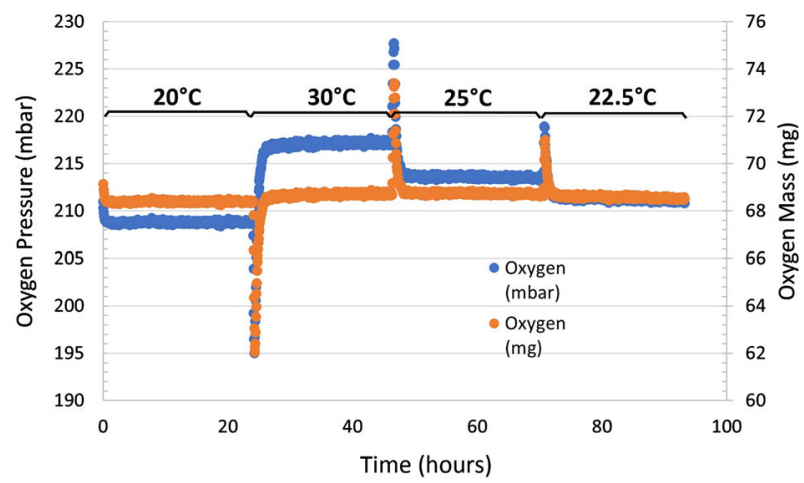

Fig. 5 The effect of changing temperature on the oxygen pressure as measured for a sealed $250 \mathrm{ml}$ Mason Ball vessel containing only air. The raw oxygen data pressure is plotted alongside the calculated oxygen mass within the vessel over time

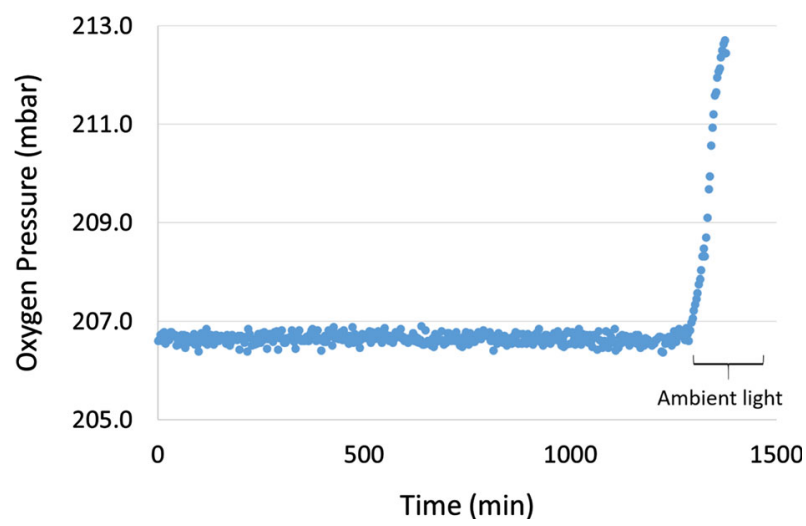

Fig. 6 The effect of ambient light on the oxygen pressure as measured for a sealed $250 \mathrm{ml}$ Mason Ball vessel containing only air. Raw oxygen pressure data are presented

could be expected that ambient light conditions in the measuring environment may influence the measurement obtained. This effect is seen in Fig. 6 where stable oxygen measurements are obtained for a sealed $250 \mathrm{ml}$ Mason Ball vessel containing air at ambient pressure in a closed climatic chamber with no ambient light. Stable values are recorded until the point at which ambient light is let into the climatic chamber, at which time the oxygen value recorded begins to climb.

Knowing that ambient light has an influence on the oxygen pressure recorded, ideally the readings would be taken without ambient light. At Cardiff, this is achieved by measuring the oxygen pressures while vessels are stored in a climate chamber with no ambient light. This will not always be achievable, however, in which case it is recommended that the lighting conditions be as stable as possible which may require the exclusion of natural light (which is changeable over time). It is also recommended that light be excluded completely from sensor spots when not being measured to avoid depletion of the sensor which affects subsequent oxygen readings [38]. It is concerning that the oxygen pressure increases with exposure time. The possibility of masking the area around the sensor spot to minimise light from passing 


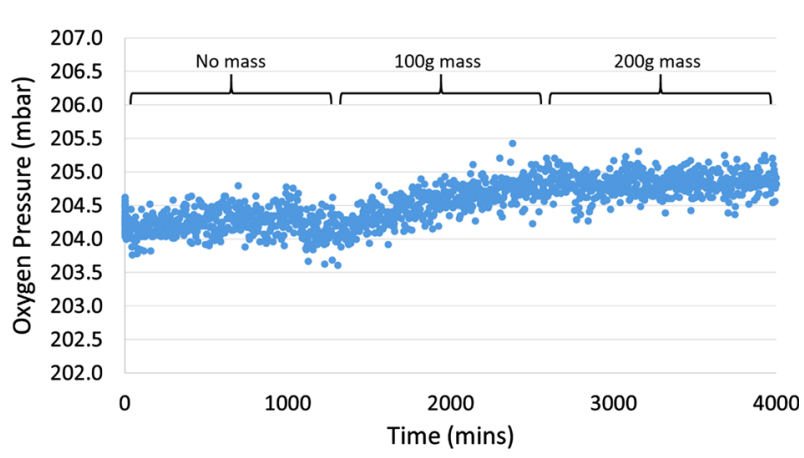

Fig. 7 The effect on oxygen pressure of a mass being placed on the reaction vessel lid as measured for a sealed $250 \mathrm{ml}$ Mason Ball vessel containing only air. Raw oxygen pressure data are presented

through the body of the glass vessel to the sensor spot could be investigated to examine whether this effect can be reduced.

\subsection{Barometric pressure}

That fluctuations in barometric pressure affect the oxygen pressure within a sealed system was shown by Matthiesen [1] for vessels sealed with a flexible barrier material such as Escal ${ }^{\mathrm{TM}}$ or aluminium foil, even where the body of the vessel was rigid. A positive correlation of increasing internal oxygen pressure with increasing barometric pressure was observed, as would be expected from a reduction in vessel volume caused by slight deformation of the flexible seal with increased external air pressure. For this reason, the Cardiff method employs rigid vessels with rigid lids to minimise these effects.

To examine the ability of these vessels to withstand deformation by atmospheric pressure on the mild steel lid (the most flexible part of the vessel), the oxygen pressure inside an empty $250 \mathrm{ml}$ Mason Ball vessel containing air at atmospheric pressure was logged for $20 \mathrm{~h}$ in a climatic chamber at stable temperature $\left(20^{\circ} \mathrm{C}\right)$ and relative humidity $(30 \%)$ before a $100 \mathrm{~g}$ copper alloy mass was placed in the centre of its lid. The oxygen pressure logging continued for a further c. $24 \mathrm{~h}$ before the $100 \mathrm{~g}$ mass was replaced with a $200 \mathrm{~g}$ mass and logging continued (Fig. 7).

The addition of a $100 \mathrm{~g}$ mass to the lid increased the oxygen pressure recorded within the vessel, likely by deformation of the lid reducing the internal volume of the vessel. Further increase in oxygen pressure on substitution of this mass for one of $200 \mathrm{~g}$ was not observed. This implies that the lid was fully depressed by the $100 \mathrm{~g}$ mass and the c. 1 mbar increase in oxygen pressure represents the extent of change likely to be seen due changes in atmospheric pressure. This small increase in oxygen pressure demonstrates the appropriateness of a rigid system for refined oxygen consumption measurement.

It is suggested that where a rigid system is not being employed, for example where samples are enclosed in Escal ${ }^{\mathrm{TM}}$, a control enclosure with no sample has its oxygen pressure recorded alongside the samples to ensure that atmospheric pressure effects can be identified and compensated for. It also highlights that depressing a flexible container with the optical fibre probe during measurement must be avoided. 


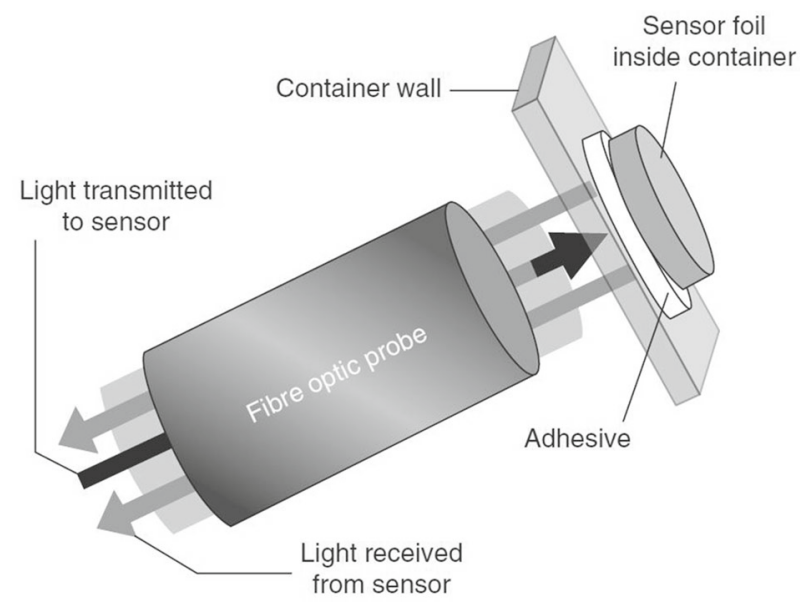

Fig. 8 Diagram showing the transmission of light between the meter and the sensor spot inside the reaction vessel. From Matthiesen [3]

\section{Fibre optic probe optimisation and amplitude}

The method of delivery of light between the meter and the sensor spot is via an optical fibre probe (Fig. 8). Experiences from experimentation at Cardiff have shown, anecdotally, that the conformation and stability of the fibre optic are important factors in obtaining high quality data. To optimise the functioning of the fibre optic:

Ensure that it has no tight bends or kinks.

Ensure that it does not vibrate during measurement.

Ensure close contact between the tip and the wall of the vessel in line with the sensor spot.

Ensure that the tip does not move in relation to the sensor spot during measuring.

Ensure that the tip is clean and free from damage.

These measures ensure that the light is delivered consistently between the meter and the sensor. The performance of the fibre optic is intimately related to a value within the measurement software, the amplitude. Amplitude is a measure of the intensity of illumination from the meter as transmitted through the fibre optic cable and is measured in microvolts $(\mu \mathrm{V})[39]$. A higher amplitude results in higher precision of readings by improving the signalto-noise ratio, and the amplitude must be at least $3000 \mu \mathrm{V}$ to take an oxygen measurement.

The relationship between amplitude and oxygen is inversely proportional (Fig. 9), but the cause and effect are complex. Experience shows that amplitude can be reduced by poor physical contact between the tip of the probe and the sensor spot, by insufficient light at source from the meter and by bleaching out of the sensor by flooding with ambient light. All of these factors will artificially raise the oxygen pressure reading due to the inverse relationship between the two parameters.

The Cardiff protocol for oxygen measurement therefore includes a verification of the amplitude when taking all measurements. For long-term corrosion rate tests during which the oxygen pressure is expected to fall, amplitude should match or exceed that of the previous measurement event in order to ensure that a low amplitude is not artificially increasing the oxygen measurement and suppressing the consumption rate. 


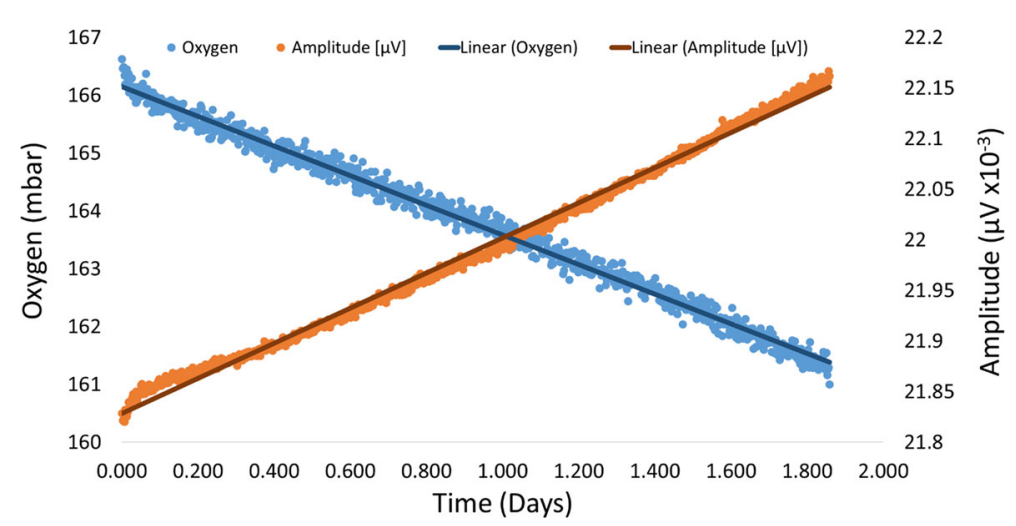

Fig. 9 The inverse relationship between oxygen pressure and amplitude of the LED signal as seen when a sample is consuming oxygen within a sealed reaction vessel. In this case, consumption of oxygen by the sample is the driver of an increasing amplitude value

\section{Measurement protocol}

The measurement protocol for an investigation will depend on the nature of the samples, the expected oxygen consumption rate and the specific data requirements. For example, samples of finely divided powder are likely to consume oxygen at a relatively fast rate unless the humidity is very low or there is a small proportion of the corrosive reactant. In these cases, the most effective method may be to log oxygen pressure continuously as the reaction proceeds. This ensures that any changes in rate due to depletion of the reactants or similar factors are captured within the data. On the other hand, samples of coated metal may be expected to have a slow corrosion rate and therefore may have their oxygen consumption charted periodically over a long timeframe [30]. The corrosion rates of archaeological samples can be highly variable, even from the same site due to intrinsic object factors [13], but are also likely to benefit from periodic measurement. This is normally achieved by logging the oxygen pressure within a vessel over a short period (e.g. 5-10 min) and averaging the oxygen value for that day.

What is important for either measurement protocol is the frequency of measurements. Over-exposure of the sensor spot can be caused by prolonged light illumination during measurement which causes an artificial decline in oxygen pressure values. The sensor spots have a recovery period following a light exposure event and logging too frequently inhibits this process [39]. This effect can be seen in Fig. 10 where logging at $1 \mathrm{~s}$ intervals causes an apparent decrease in oxygen pressure within an empty vessel at static external temperature and humidity within a closed climatic chamber. When the measurement interval is increased to once every $3 \mathrm{~min}$, the sensor spots exhibit a slow recovery until the oxygen values match the starting values.

This shows the importance of not over-exposing the sensor spots. For a continuously logging protocol, the Cardiff method recommends a 3- or 5-min logging interval. For longterm investigations with periodic measurement, logging at $30 \mathrm{~s}$ intervals for 5-10 min will not deplete the sensor spot. 


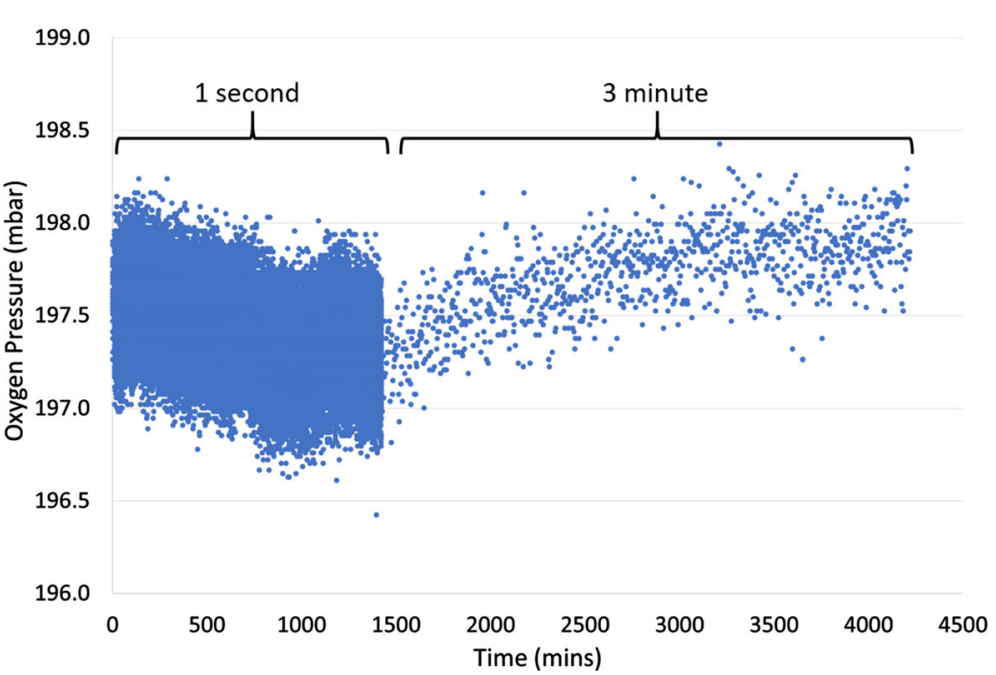

Fig. 10 The effect of measurement frequency on oxygen pressure as measured for a sealed $250 \mathrm{ml}$ Mason Ball vessel containing only air. The initial decline when measuring at $1 \mathrm{~s}$ interval shows photoluminescent decay of the sensor spot which recovers as measurement frequency is reduced to 3 min intervals

\section{Calculating loss of metal during corrosion}

\subsection{Method}

Methods of converting oxygen consumption values and rates into projections of loss of metallic substance from artefacts over time have been reported in several publications $[3,15$, 34]. For wrought iron, for example, this follows the assumption that the predominant reaction occurring in atmospheric corrosion is as follows:

$$
4 \mathrm{Fe}+3 \mathrm{O}_{2}+2 \mathrm{H}_{2} \mathrm{O} \rightarrow 4 \mathrm{FeOOH}
$$

Therefore, the ratio of reacting moles of oxygen and iron can be used to calculate the moles of metallic iron converted during corrosion according to the recorded moles of oxygen consumed. This can be further related to the surface area of the sample to estimate the loss of depth of iron over time due to corrosion processes.

\subsection{Considerations}

The value of performing these calculations must be scrutinised, and there are impediments to their application to heritage metals. Calculations that are reliant on measuring the free air space in a reaction vessel are challenging to follow for some samples as the porosities of corrosion product layers are difficult to calculate. Although this can be achieved by immersion in a fluid for some artefacts, this will not be acceptable for all. Similarly, calculating the surface area of the reactive metal core for a corroded object which is covered in voluminous corrosion products and is of irregular shape, can be impossible without appropriate technology. Additionally, the controlled environment within a reaction vessel is an 'unreal' scenario for many heritage artefacts. Calculations also assume that all oxygen consumption recorded is involved in the corrosion reaction rather than any other processes. 
Assuming that the calculations can be made and the values obtained are reliable, what do they mean for heritage artefacts? Study has shown that the corrosion of chloride-infested archaeological iron, for example, is not a process that occurs evenly across the metal surface [40]. Instead, it is localised in pits and this localised corrosion can cause catastrophic damage and break-up of an artefact long before the metal core has been converted by corrosion processes. In this way, the complete loss of heritage value of an artefact may not be related to predictions of loss of metal over time and oxygen consumption cannot provide the answer to all aspects of artefact stability.

\section{Conclusion}

From its introduction to heritage science more than a decade ago, oxygen consumption as a proxy for corrosion rate measurement of metals has become an important tool for understanding the environmentally induced decay of artefacts and the efficacy of conservation treatments. The refinement of the technique for different applications within the field has taken time, experimentation and experience.

\subsection{Guidance for heritage scientists}

This paper has presented details of the Cardiff protocol for application of oxygen consumption to various sample types based on experience of its use. To summarise the guidance:

- Choose a transparent reaction vessel of an appropriate size (volume and aperture) to accommodate the required sample and buffer material to control relative humidity.

- Choose a rigid reaction vessel with rigid lid to reduce the likelihood of barometric pressure influencing the oxygen measurements.

- Verify the leakage rates of vessels by filling with nitrogen and recording ingress of oxygen over time.

- Ensure that no components of your system consume oxygen by measuring oxygen pressure in a sealed vessel containing all components apart from the sample.

- If possible, stabilise the environment around the reaction vessel during oxygen measurement, particularly temperature and ambient light, and ensure that they are the same each time a vessel is measured. If it is not possible to stabilise temperature, ensure that short-term fluctuations are recorded for use in calculations and be aware that data may be affected.

- Inspect the fibre optic probe and ensure that its tip is free from damage and there are no kinks in its length.

- Ensure good contact between the fibre optic tip and the vessel wall and good alignment with the sensor spot.

- Ensure that there is no vibration of the fibre optic cable during measurement.

- Check the amplitude at the beginning of each measurement and ensure that it matches or is higher than that of the previous measurement (for samples expected to consume oxygen).

- Protect sensor spots from light-induced damage by storing in the dark and covering with blackout material when not being measured.

- Design a measurement frequency protocol that is appropriate for the sample type and does not damage the sensor spot.

- Consider the data and its applicability to heritage contexts when performing calculations and drawing conclusions. 


\subsection{Limitations and ongoing research}

The use of rigid vessels can be challenging for heritage artefacts of different sizes with lack of availability of a full range of airtight vessels. This is a limitation of the Cardiff method, particularly for application to large samples and artefacts. Ongoing work aims to identify further methods of creating airtightness in different vessels by sealing to increase flexibility in sample type whilst maintaining control of influential parameters. Another disadvantage is the relatively long data collection periods for samples with slow oxygen consumption rates. The reduction in this time by occupation of headspace with inert materials will be investigated.

In summary, oxygen consumption is an extremely useful tool for assessing the stability of metals, but there is more to be discovered about application of the technique and extrapolation of its results to management of heritage materials.

Funding AHRC/EPSRC Science and Heritage AH/H032754/1.

Data Availability Statement This manuscript has no associated data or the data will not be deposited. [Authors' comment: There are no associated data available.]

Open Access This article is licensed under a Creative Commons Attribution 4.0 International License, which permits use, sharing, adaptation, distribution and reproduction in any medium or format, as long as you give appropriate credit to the original author(s) and the source, provide a link to the Creative Commons licence, and indicate if changes were made. The images or other third party material in this article are included in the article's Creative Commons licence, unless indicated otherwise in a credit line to the material. If material is not included in the article's Creative Commons licence and your intended use is not permitted by statutory regulation or exceeds the permitted use, you will need to obtain permission directly from the copyright holder. To view a copy of this licence, visit http://creativecommons.org/licenses/by/4.0/.

\section{References}

1. H. Matthiesen, A novel method to determine oxidation rates of heritage materials in vitro and in situ. Stud. Conserv. 52, 271-280 (2007)

2. H. Matthiesen, K. Wonsyld, In situ measurement of oxygen consumption to estimate corrosion rates. Corros. Eng., Sci. Technol. 45(5), 350-356 (2010)

3. Matthiesen, H., Oxygen monitoring in the corrosion and preservation of metallic heritage artefacts. Corrosion and conservation of cultural heritage artefacts, 2013: p. 368-391.

4. Thickett, D., S. Lambarth, and P. Wyeth. Determining the stability and durability of archaeological materials. in 9th International Conference on NDT of Art, Jerusalem, Israel. 2008.

5. N.A. North, C. Pearson, Washing methods for chloride removal from marine iron artifacts. Stud. Conserv. 23(4), 174-186 (1978)

6. North, N.A. and C. Pearson, Alkaline sulfite reduction treatment of marine iron, in ICOM Committee for Conservation 4th triennial meeting, Venice, 13-18 October 1975: preprints, I.C.f. Conservation, Editor. 1975, International Council of Museums: Paris, France. pp. 75133-1-75133-14.

7. N.A. North, C. Pearson, Investigations into methods for conserving iron relics recovered from the sea, in Conservation in archaeology and the applied arts: preprints of the contributions to the Stockholm Congress, 2-6 June 1975. (International Institute for Conservation of Historic and Artistic Works, London, United Kingdom, 1975), pp. 173-181

8. S. Keene, C. Orton, Stability of treated archaeological iron: an assessment. Stud. Conserv. 30(3), 136-142 (1985)

9. W.A. Oddy, J. Black. A new method for the conservation of iron: ionophoresis in a non-aqueous electrolyte. in Recent advances in the conservation and analysis of artifacts. Jubilee Conservation Conference, London 6-10 July 1987. 1987.

10. M. Rimmer, D. Watkinson, Q. Wang, The efficiency of chloride extraction from archaeological iron objects using deoxygenated alkaline solutions. Stud. Conserv. 57(1), 29-41 (2012)

11. D. Watkinson, M. Rimmer, Quantifying Effectiveness of chloride desalination treatments for archaeological iron using oxygen measurement, in Metal 2013 Edinburgh, Scotland. Interim meeting of the 
international Council of Museums Committee for Conservation Metal Working Group, E. Hyslop, Gonzalez, Vanesa, Troalen, Lore and Wilson, Lyn, ed. 2013, Historic Scotland: Edinburgh, UK. pp. 95-102.

12. D. Watkinson, N. Emmerson, J. Seifert, Matching Display Relative Humidity to Corrosion Rate: Quantitative Evidence for Marine Cast Iron Cannon Balls, in Metal 2016 New Delhi, India. Interim meeting of the international Council of Museums Committee for Conservation Metal Working Group. 2016: New Delhi, India.

13. D.E. Watkinson, M.B. Rimmer, N.J. Emmerson, The Influence of Relative Humidity and Intrinsic Chloride on Post-excavation Corrosion Rates of Archaeological Wrought Iron. Studies in Conservation, 2019: pp. $1-16$.

14. J.C. Thunberg, D.E. Watkinson, and N.J. Emmerson, Desiccated Microclimates for Heritage Metals: Creation and Management. Studies in Conservation, 2020: pp. 1-27.

15. N.J. Emmerson, D.E. Watkinson, Surface preparation of historic wrought iron: evidencing the requirement for standardisation. Mater. Corros. 67(2), 176-189 (2016)

16. N. Emmerson, D. Watkinson, and J. Thunberg, Flame cleaning of historic wrought iron: practitioner methods and their impact on oxide morphologies and post-treatment corrosion rates, in Metal 2019 Neuchâtel, Switzerland. Interim meeting of the International Council of Museums Committee for Conservation Metal Working Group, C. Chemello, L. Brambilla, and E. Joseph, eds. 2019, Haute Ecole Arc Conservation-restauration (HE-Arc CR) \& International Council of Museums - Committee for Conservation (ICOM-CC): Neuchâtel, Swizterland. pp. 281-288.

17. D. Watkinson, M. Lewis, Great Britain iron hull: modelling corrosion to define storage relative humidity. Metal 4, 88-103 (2004)

18. D. Watkinson, M.R.T. Lewis, Desiccated storage of chloride-contaminated archaeological iron objects. Stud. Conserv. 50(4), 241-252 (2005)

19. M. Rimmer, D. Watkinson, Residues of alkaline sulphite treatment and their effects on the corrosion of archaeological iron objects, in Metal 2010: proceedings of the interim meeting of the ICOM-CC Metal Working Group, October 11-15, 2010, Charleston, South Carolina, USA, P. Mardikian, et al., Editors. 2011, Clemson University: Clemson, South Carolina, United States. pp. 16-23.

20. M. Rimmer, D. Watkinson, Q. Wang, The impact of chloride desalination on the corrosion rate of archaeological iron. Stud. Conserv. 58(4), 326-337 (2013)

21. D. Thickett, M. Odlyha, The formation and transformation of akaganeite, in Metal 2013 Edinburgh, Scotland. Interim meeting of the international Council of Museums Committee for Conservation Metal Working Group, E. Hyslop, Gonzalez, Vanesa, Troalen, Lore and Wilson, Lyn, Editor. 2013, Historic Scotland: Edinburgh, UK. pp. 103-109.

22. E. Nordgren, The effect of metallurgical structure on the chloride-induced corroion of archaeological wrought iron, in Department of Archaeology and Conservation. 2016, Cardiff University: Cardiff University. p. 294.

23. D.E. Watkinson, N.J. Emmerson, The impact of aqueous washing on the ability of $\beta \mathrm{FeOOH}$ to corrode iron. Environ. Sci. Pollut. Res. 24(3), 2138-2149 (2017)

24. B. Schmutzler, Z. Revay, C. Stieghorst, Desalination of Archaeological Iron Objects: Comparing the Effectiveness of Sodium Hydroxide Treatments, in Metal 2019 Neuchâtel, Switzerland. Interim meeting of the International Council of Museums Committee for Conservation Metal Working Group, C. Chemello, L. Brambilla, and E. Joseph, Editors. 2019, Haute Ecole Arc Conservation-restauration (HE-Arc CR) \& International Council of Museums - Committee for Conservation (ICOM-CC): Neuchâtel, Swizterland. pp. 250-256.

25. H. Kautsky, Quenching of luminescence by oxygen. Trans. Faraday Soc. 35, 216-219 (1939)

26. J.R. Lakowicz, Principles of Fluorescence Spectroscopy (Springer Science \& Business Media, New York, 2013).

27. H.C. Bittig et al., Oxygen optode sensors: principle, characterization, calibration, and application in the ocean. Front. Mar. Sci. 4, 429 (2018)

28. PreSens, Oxygen Sensor Spots PSTt3/PSt6 Instruction Manual. 2017, Precision Sensing GmbH: Regensburg, Germany.

29. H. Matthiesen, K. Stemann-Petersen. A fast and non-destructive method to document and quantify the efficiency of metals conservation. in Metal 2013: Interim Meeting of the ICOM-CC Metal Working Group. Edinburgh. 2013.

30. N. Emmerson, Heritage wrought iron: towards the development of evidence based standards for coating. 2015, Cardiff University.

31. A. Lawson, Assessment of the performance of three clear coatings for use in heritage conservation by an oxygen consumption technique. 2016, Cardiff University. p. 295.

32. S. Turgoose, The corrosion of archaeological iron during burial and treatment. Stud. Conserv. 30(1), 13-18 (1985) 
33. S. Turgoose, Post-Excavation Changes in Iron Antiquities. Stud. Conserv. 27(3), 97-101 (1982)

34. N. Emmerson, D. Watkinson, Preparing historic wrought iron for protective coatings: quantitative assessment to produce evidence-based protocols, in Metal 2013 Edinburgh, Scotland. Interim meeting of the international Council of Museums Committee for Conservation Metal Working Group, E. Hyslop, Gonzalez, Vanesa, Troalen, Lore and Wilson, Lyn, Editor. 2013, Historic Scotland: Edinburgh, UK.

35. D. Thickett, Critical relative humidity levels and carbonyl pollution concentrations for archaeological copper alloys. Proc. ICOM-CC Metals Work. Group New Delhi 2016, 180-187 (2016)

36. S. Weintraub, Demystifying silica gel. Object Specialty Group, 2002.

37. H. Golnabi, Using three different optical fiber designs to study humidity effect on the air refractive index. Opt. Lasers Eng. 50(11), 1495-1500 (2012)

38. P. Hartmann, M.J.P. Leiner, P. Kohlbacher, Photobleaching of a ruthenium complex in polymers used for oxygen optodes and its inhibition by singlet oxygen quenchers. Sens. Actuators, B Chem. 51(1), 196-202 (1998)

39. PreSens, PreSens Measurement Studio 2 for O2 Instruction Manual. 2018, Precision Sensing GmbH: Regensburg, Germany. p. 70.

40. D. Watkinson et al., The use of neutron analysis techniques for detecting the concentration and distribution of chloride ions in archaeological iron. Archaeometry 56(5), 841-859 (2014) 